\title{
Joint Channel Estimation and Co-Channel Interference Cancellation in Wireless Networks Using Belief Propagation
}

\author{
Yan Zhu, Dongning Guo and Michael L. Honig \\ Department of Electrical Engineering and Computer Science \\ Northwestern University, Evanston, Illinois 60208
}

\begin{abstract}
This paper studies signal detection in wireless networks where the uncertainty is due to the fading channel as well as strong co-channel interference of the same form as that of the desired signal. In particular, unlike for the desired signal, no pilot for the interference signal is available for measuring its fading channel state. Still, the interference is a non-Gaussian process and treating it as Gaussian noise can lead to poor performance. We propose a joint channel estimation and interference cancellation scheme based on belief propagation, which is capable of fully exploiting the statistics of the interference. Simulation results show that the receiver performs significantly better compared to conventional receivers with linear channel estimation.
\end{abstract}

\section{INTRODUCTION}

Wireless networks are fundamentally limited by two factors, namely, uncertainties about the time-varying communication channel and interference from other terminals in the system. Although each of the two issues has been studied in depth assuming the absence of the other one, much less is understood about the situations where both factors are significant.

This work considers the detection of one signal in a time varying fading channel, which is also subject to interference signals of the same form and possibly of similar strength. Such problems arise for example in cellular networks where co-channel interference is strong. Another example is ad hoc networks where terminals inevitably interfere with each other.

For simplicity, we restrict our attention to a two-user, narrow-band system with BPSK modulation, where the fading channel of each user is modeled as a Gauss-Markov process. Besides practical usage on its own, the model is the elementary building block of orthogonal frequency division multiplexing (OFDM), which is the basis for next generation wireless systems. We assume knowledge of the fading statistics. ${ }^{1}$ We also assume that the desired user's channel can be measured using known pilot symbols interleaved with data symbols, while no pilot is available for the interferer. This is a typical scenario in wireless systems where the pilot pattern of the cochannel interference signal is unavailable, e.g., in a cellular system where the interference is from a different cell than that of the desired user.

The detection problem is challenging due to simultaneous uncertainties of the channels as well as the interference. Con-

This work is supported in part by the Northwestern Motorola Center for Seamless Communications.

${ }^{1}$ In principle, the statistics are not needed a priori and can be estimated. ventionally, the channel state is measured assuming absence of interference, and interference is suppressed assuming complete knowledge about the channel state. In particular, as is seen in [9], knowledge of interfering pilots is indispensable to the success of linear channel estimation, even with iterative Turbo processing. The reason is that linear estimators treat the interference process as white Gaussian noise. If the interference is strong compared to the desired signal, the signal-to-noiseand-interference ratio is small, which leads to unacceptable channel estimation performance.

In this work, we propose a general approach to joint channel estimation and interference cancellation in multiuser systems, which can fully exploit knowledge of the non-Gaussian statistics of the interference. Consider the detection of a sequence of symbols of the desired user with one strong interferer of the same signaling format, where the fading processes of both the desired user and the interference are Gauss-Markov. The problem is fundamentally a compound hypothesis testing problem (averaged over channel uncertainty). Unfortunately, the maximum likelihood sequence detector, which exhausts the exponential number of hypotheses, is computationally prohibitive.

The low-complexity scheme proposed in this paper is based on belief propagation (BP), which is an iterative messagepassing algorithm for performing statistical inference on graphical models by propagating locally computed "beliefs" [5]. One of the most successful applications of BP is the decoding of low-density parity-check (LDPC) codes [7], [8]. Applications of BP to receiver design can be found in [3], [4], where the authors consider a single user in fading channels. It turns out that the problem at hand is equivalent to statistical inference on a tree-like graph, for which BP is ultimately optimal. For implementation, BP has to be constrained both in the number of iterations and in the way the messages exchanged between vertices in the graph are described. As numerical results in this paper demonstrate, the resulting suboptimal scheme performs remarkably better than the usual techniques of linear channel estimation followed by detection of individual symbols.

\section{SYSTEM MODEL}

Consider an uncoded narrow-band system, where the received signal at time $i$ in a frame (or block) of length $n$ is 
expressed as

$$
Y_{i}=H_{i} X_{i}+H_{i}^{\prime} X_{i}^{\prime}+N_{i} \quad i=1 \ldots n
$$

where $X_{i}$ and $X_{i}^{\prime}$ denote the transmitted symbols of the desired user and interferer respectively, $H_{i}$ and $H_{i}^{\prime}$ denote the corresponding channel gains, and $\left\{N_{i}\right\}$ is the sequence of i.i.d. circularly-symmetric complex Gaussian noise with zero mean and variance $\sigma_{N}^{2}$. For simplicity, we assume BPSK modulation, i.e., $X_{i}, X_{i}^{\prime}= \pm 1$.

Assuming Rayleigh fading, $\left\{H_{i}\right\}$ and $\left\{H_{i}^{\prime}\right\}$ are modeled as two independent Gauss-Markov processes, i.e., they are generated by first-order auto-regressive systems (e.g., [1]):

$$
\begin{aligned}
& H_{i}=\alpha H_{i-1}+\sqrt{1-\alpha^{2}} W_{i} \\
& H_{i}^{\prime}=\alpha H_{i-1}^{\prime}+\sqrt{1-\alpha^{2}} W_{i}^{\prime}
\end{aligned}
$$

where $W_{i}$ and $W_{i}^{\prime}$ are two independent white Gaussian processes with variance $\sigma_{H}^{2}$ and $\sigma_{H}^{\prime 2}$, respectively, and $\alpha$ is the correlation coefficient between adjacent fading coefficients. Note that $\alpha=0$ corresponds to independent fading, while $H_{i}$ and $H_{i}^{\prime}$ becomes static if $\alpha=1$, which corresponds to block fading. Note that (1) also models an OFDM system where $i$ denotes the index of sub-carriers instead of time index. More generally, $i$ may index symbols in the time-frequency grid in OFDM systems experiencing both time and frequency selective channels.

Typically, pilots are inserted periodically between data symbols. For example, the pilot pattern "PDDDPDDDPDDD..." denotes $25 \%$ pilots, where $\mathrm{P}$ and $\mathrm{D}$ mark pilot and data symbols respectively. Let $Y_{i}^{j}$ denote the sequence $Y_{i}, Y_{i+1}, \ldots, Y_{j}$. The detection problem can be formulated as follows: Given the observations $Y_{1}^{n}$ and the small subset of known pilots in $X_{1}^{n}$, we would like to detect the information symbols from desired user, i.e., the remaining unknown symbols in $X_{1}^{n}$. Note that no knowledge of the channels and interfering symbols are available except for their statistics.

\section{GRAPHICAL MODEL AND BP ALGORITHM}

\section{A. Graphical Model}

An important observation from (1) and (2) is that the fading coefficients $\left(H_{i}, H_{i}^{\prime}\right)$ form a Markov chain with state space in $\mathbb{C}^{2}$. Also, given $\left\{\left(H_{i}, H_{i}^{\prime}\right)\right\}_{i=1}^{n}$, the input and output variables as a 3-tuple $\left(X_{i}, X_{i}^{\prime}, Y_{i}\right)$ at each time instant $i$, is independent over time $i=1,2, \ldots, n$. Therefore, the relationships (1) and (2) can be described using a factor graph as is shown in Fig. 1.

Generally, a factor graph is a bipartite graph, which consists of two types of nodes: the variable nodes, denoted by circles in the graph, which represent the variables, and the factor nodes, denoted by squares which represent the constraints among the variable nodes connected to them. For example, the node labeled as " $H_{i}, H_{i}^{\prime}$ " represents the pair of random variables $\left(H_{i}, H_{i}^{\prime}\right)$. The factor node connecting nodes $Y_{i},\left(H_{i}, H_{i}^{\prime}\right)$, and $\left(X_{i}, X_{i}^{\prime}\right)$ represents the probabilistic constraint among them, which is specified by (1) and is equivalent to the conditional

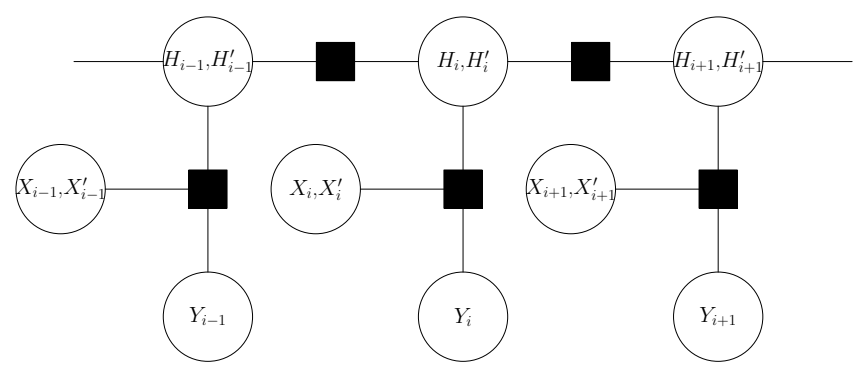

Fig. 1: A factor graph describing the two-user system.

Gaussian probability density $p\left(y_{i} \mid h_{i}, h_{i}^{\prime}, x_{i}, x_{i}^{\prime}\right)$. Similarly, the factor node between nodes $\left(H_{i}, H_{i}^{\prime}\right)$ and $\left(H_{i-1}, H_{i-1}^{\prime}\right)$ represents the relationship given by (2), i.e., $p\left(h_{i}, h_{i}^{\prime} \mid h_{i-1}, h_{i-1}^{\prime}\right)$. The prior probability distribution of the data symbols is assigned as follows. All BPSK symbols $X_{i}$ and $X_{i}^{\prime}$ are uniformly distributed on $\{-1,1\}$ except for the subset of pilot symbols in $X_{1}^{n}$, for which $P\left\{X_{i}=1\right\}=1$. The Markovian property of the graph is that conditioned on any cut node(s), the separated subsets of variables are mutually independent. As we shall see, the Markovian property plays an important role in the development of the message passing algorithm.

Since the graphical model in Fig. 1 fully describes the probability laws of the random variables given by (1) and (2), the detection problem is equivalent to statistical inference on the graph. Simply put, we seek to answer the following question: Given the realization of a subset of the variables on the graph, what can be inferred about the remaining variables?

\section{B. Exact Inference Via Message Passing}

In the detection problem described in Section II, the goal of inference is to obtain the marginal posterior probability $p\left(x_{i} \mid y_{1}^{n}\right)$ for all $i$ which corresponds to unknown symbols. Problems of such nature have been widely studied (see e.g., [6, Chapter 4] and [5]). In particular, belief propagation is an efficient algorithm for computing the posteriors by iteratively passing messages between neighboring nodes on the graph. In principle, the result of message passing with sufficient iterations gives the exact a posteriori probability of each unknown random variable if the factor graph is a tree (i.e., free of cycles) as is the case in the problem at hand. For general graphs with cycles, the result of BP is an approximation.

For notational convenience, let $G_{i}=\left[H_{i}, H_{i}^{\prime}\right]^{\mathrm{T}}$ and $Z_{i}=$ $\left[X_{i}, X_{i}^{\prime}\right]^{\mathrm{T}}$. The goal is to compute for each $i=1, \ldots, n$ :

$$
\begin{aligned}
p\left(x_{i} \mid y_{1}^{n}\right) & =\sum_{x_{i}^{\prime}} \int p\left(z_{i}, g_{i} \mid y_{1}^{n}\right) \mathrm{d} g_{i} \\
& \propto \sum_{x_{i}^{\prime}} \int p\left(z_{i}, g_{i}, y_{1}^{n}\right) \mathrm{d} g_{i}
\end{aligned}
$$

where we use the "proportion" notation $\propto$. This is because $Y_{1}^{n}=y_{1}^{n}$ is observed, and so that its probability is irrelevant to the inference problem and can be treated as a constant. By the Markovian property, $Z_{i}, Y_{i}, Y_{1}^{i-1}$ and $Y_{i+1}^{n}$ are mutually 
independent given $G_{i}$. Therefore,

$$
\begin{aligned}
p\left(x_{i} \mid y_{1}^{n}\right) & \propto \sum_{x_{i}^{\prime}} \int p\left(y_{i}, z_{i} \mid g_{i}\right) p\left(y_{1}^{i-1} \mid g_{i}\right) p\left(y_{i+1}^{n} \mid g_{i}\right) p\left(g_{i}\right) \mathrm{d} g_{i} \\
& \propto \sum_{x_{i}^{\prime}} \int p\left(y_{i}, z_{i} \mid g_{i}\right) p\left(g_{i} \mid y_{1}^{i-1}\right) p\left(g_{i} \mid y_{i+1}^{n}\right) / p\left(g_{i}\right) \mathrm{d} g_{i}
\end{aligned}
$$

Since $Z_{i}$ and $G_{i}$ are independent, we finally have

$$
p\left(x_{i} \mid y_{1}^{n}\right) \propto \sum_{x_{i}^{\prime}} p\left(z_{i}\right) \int p\left(y_{i} \mid g_{i}, z_{i}\right) p\left(g_{i} \mid y_{1}^{i-1}\right) p\left(g_{i} \mid y_{i+1}^{n}\right) / p\left(g_{i}\right) \mathrm{d} g_{i}
$$

Note that $p\left(y_{i} \mid g_{i}, z_{i}\right)$ can be obtained by (1) and $p\left(z_{i}\right)=$ $p\left(x_{i}\right) p\left(x_{i}^{\prime}\right)$ since symbols from the two users are independent. Also, $P\left(X_{i}=1\right)=1-P\left(X_{i}=-1\right)=1$ if the $i$ th symbol is the pilot for the desired user, otherwise $P\left(X_{i}=1\right)=$ $P\left(X_{i}=-1\right)=1 / 2$. Moreover, $p\left(x_{i}^{\prime}\right) \equiv 1 / 2$ for all $i$, since we do not know the pilot pattern of the interfering user. In order to compute (3), it is sufficient to compute $p\left(g_{i} \mid y_{1}^{i-1}\right)$ and $p\left(g_{i} \mid y_{i+1}^{n}\right)$, respectively.

We give a brief derivation of $p\left(g_{i} \mid y_{i+1}^{n}\right)$ in below, whereas computation of $p\left(g_{i} \mid y_{1}^{i-1}\right)$ is straightforward by symmetry. The technique is to develop a recursion for the posterior probability. First, we have

$$
p\left(g_{i} \mid y_{i+1}^{n}\right)=\int p\left(g_{i} \mid g_{i+1}\right) p\left(g_{i+1} \mid y_{i+1}^{n}\right) \mathrm{d} g_{i+1}
$$

because given $G_{i+1}, G_{i}$ and $Y_{i+1}^{n}$ are independent. By the Markovian property, $Y_{i+2}^{n}$ and $Y_{i+1}$ are independent given $G_{i+1}$. Therefore,

$$
p\left(g_{i} \mid y_{i+1}^{n}\right) \propto \int p\left(g_{i} \mid g_{i+1}\right) p\left(y_{i+1} \mid g_{i+1}\right) p\left(g_{i+1} \mid y_{i+2}^{n}\right) \mathrm{d} g_{i+1}
$$

Since $G_{i+1}$ and $Z_{i+1}$ are independent,

$$
\begin{aligned}
p\left(y_{i+1} \mid g_{i+1}\right) & =\sum_{z_{i+1}} p\left(y_{i+1}, z_{i+1} \mid g_{i+1}\right) \\
& =\sum_{z_{i+1}} p\left(y_{i+1} \mid z_{i+1}, g_{i+1}\right) p\left(z_{i+1}\right)
\end{aligned}
$$

Therefore, we have

$$
\begin{aligned}
& p\left(g_{i} \mid y_{i+1}^{n}\right) \propto \sum_{z_{i}} \int p\left(g_{i} \mid g_{i+1}\right) p\left(g_{i+1} \mid y_{i+2}^{n}\right) \\
& p\left(y_{i+1} \mid g_{i+1}, z_{i+1}\right) p\left(z_{i}\right) \mathrm{d} g_{i+1}
\end{aligned}
$$

Note that $p\left(g_{i} \mid g_{i+1}\right)$ can be obtained from (2). Therefore, (4) gives a recursion for computing $p\left(g_{i} \mid y_{i+1}^{n}\right)$ for each $i=1, \ldots, n$, which is the essence of the message passing algorithm. The joint channel estimation and interference cancellation algorithm is summarized in Algorithm 1.

Basically, the message from a factor node to a variable node is a summary of knowledge about the random variable(s) represented by the variable node based on all observations connected directly or indirectly to the factor node. For example, the message received by node $\left(H_{i}, H_{i}^{\prime}\right)$ from the factor node on its right side summarizes all the information about $\left(H_{i}, H_{i}^{\prime}\right)$ based on the observations $Y_{i+1}, \ldots, Y_{n}$, which is proportional

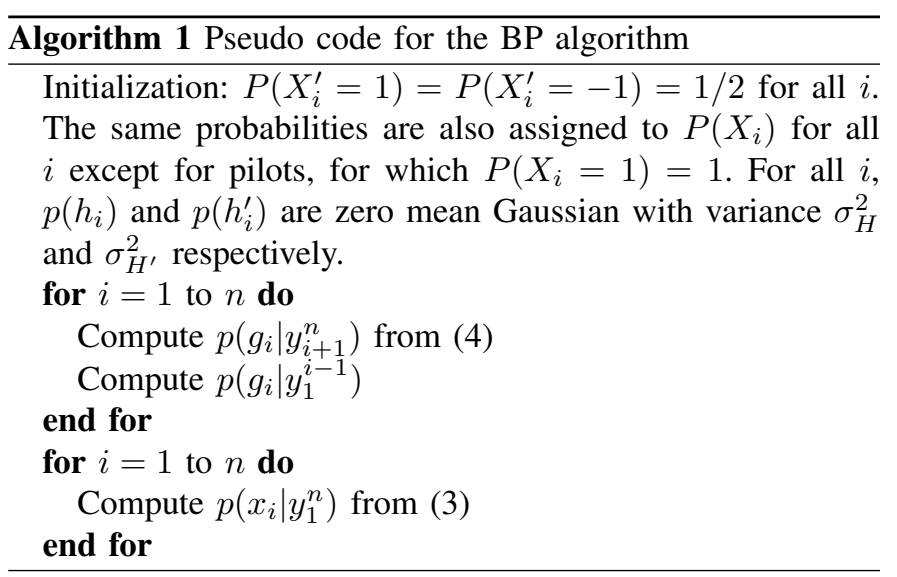

to $p\left(h_{i}, h_{i}^{\prime} \mid y_{i+1}^{n}\right)$. The message from a variable node to a factor node is a summary of information about the variable node based on the observations connected to it. For example, the message passed by node $\left(H_{i}, H_{i}^{\prime}\right)$ to the factor node on its left is the inference about $\left(H_{i}, H_{i}^{\prime}\right)$ based on the observations $Y_{i}, \ldots, Y_{n}$, i.e., $p\left(h_{i}, h_{i}^{\prime} \mid y_{i}^{n}\right)$.

\section{Implementation Issues}

Algorithm 1 cannot be implemented directly using a digital computer because the messages are continuous probability density functions (PDF). We therefore need a way to parameterize the PDFs.

An observation is that the random variables in Fig. 1 are either Gaussian or discrete. According to (4), it can be shown by induction that the density functions, $p\left(g_{i} \mid y_{i+1}^{n}\right)$ and $p\left(g_{i} \mid y_{1}^{i-1}\right)$ are mixture Gaussian functions. The Gaussian mixture function is completely characterized by the amplitude, mean and variance of each component. Therefore, we can compute and pass these parameters only. Let

$$
\mathcal{C N}(\mathbf{x}, \mathbf{m}, \mathbf{K}) \equiv \frac{1}{\pi^{l} \operatorname{det}(\mathbf{K})} \exp \left(-(\mathbf{x}-\mathbf{m})^{\mathrm{H}} \mathbf{K}^{-1}(\mathbf{x}-\mathbf{m})\right)
$$

where $m_{l \times 1}$ and $K_{l \times l}$ denote the mean and covariance, respectively. Then we can write $p\left(g_{i} \mid g_{i+1}\right)=\mathcal{C N}\left(g_{i}, \alpha g_{i+1}, B\right)$, $p\left(g_{i}\right)=\mathcal{C N}\left(g_{i}, 0, Q\right) \quad$ and $\quad p\left(y_{i} \mid g_{i}, z_{i}\right)=\mathcal{C N}\left(y_{i}, z_{i}^{\mathrm{T}} g_{i}, \sigma_{N}^{2}\right)$, where $Q=\operatorname{diag}\left(\sigma_{H}^{2}, \sigma_{H^{\prime}}^{2}\right)$ and $B=\left(1-\alpha^{2}\right) Q$.

Note that the message passing starts with a Gaussian density function and, according to (4), as the message is passed, it becomes a mixture of more and more Gaussian densities. Therefore, without loss of generality, we assume that $p\left(g_{i+1} \mid y_{i+2}^{n}\right)=\sum_{j} \rho_{j} \mathcal{C N}\left(g_{i+1}, m_{j}, K_{j}\right)$. Substituting into (4), we have after some manipulations

$$
p\left(g_{i} \mid y_{i+1}^{n}\right) \propto \sum_{j} \sum_{z_{i+1}} \rho_{j} p\left(z_{i+1}\right) L\left(j, z_{i+1}\right) C\left(j, z_{i+1}\right)
$$

where

$$
L\left(j, z_{i+1}\right)=\mathcal{C N}\left(z_{i+1}^{\mathrm{T}} m_{j}, y_{i+1}, \sigma_{N}^{2}+z_{i+1}^{\mathrm{T}} K_{j} z_{i+1}^{*}\right)
$$


and

$$
\begin{array}{r}
C\left(j, z_{i+1}\right)=\mathcal{C N}\left(g_{i}, \alpha\left[\frac{K_{j} z_{i+1}^{*}}{\sigma_{N}^{2}+z_{i+1}^{\mathrm{T}} K_{j} z_{i+1}^{*}}\left(y_{i+1}-z_{i+1}^{\mathrm{T}} m_{j}\right)\right.\right. \\
\left.\left.+m_{j}\right],\left(\Lambda_{j}^{-1}+\frac{1}{\sigma_{N}^{2}+z_{i+1}^{\mathrm{T}} \Gamma_{j} z_{i+1}^{*}} f_{j}^{\mathrm{H}} f_{j}\right)^{-1}\right)
\end{array}
$$

where $\Lambda_{j}=\alpha^{2} K_{j}+B, \Gamma_{j}=K_{j} \Lambda_{j}^{-1} B$ and $f_{j}=$ $\alpha z_{i+1}^{\mathrm{T}} K_{j} \Lambda_{j}^{-1}$. Basically, (5), (6) and (7) give an explicit recursive computation for the amplitude, mean and variance of each Gaussian component in message $p\left(g_{i} \mid y_{i+1}^{n}\right)$. Similar computations apply for $p\left(g_{i} \mid y_{1}^{i-1}\right)$. Therefore, the BP algorithm requires message-passing backward and forward once each direction, which is similar to the BCJR algorithm [2]. The key difference between our algorithm and BCJR is that the Markov chain here has a continuous state space.

The preceding Gaussian mixture representation can also be used to compute $p\left(g_{i} \mid y_{i+1}^{n}\right), p\left(g_{i} \mid y_{1}^{i-1}\right)$ and $p\left(g_{i} \mid y_{1}^{n}\right)$. The number of Gaussian components increases exponentially in the recursive formula (5), which is intractable in practice. One way to deal with this issue is to merge some of the components in order to keep a fixed number of components. In this work, we simply keep the components with the largest amplitudes. Of course, other schemes may also be used to approximate a mixture Gaussian function with fewer components.

By restricting the number of components in each message, the complexity of the algorithm is linear in the block length $n$, i.e., it is a constant per symbol. One can trade complexity with performance, in the sense that if the message is unrestricted, then the algorithm implements the maximum $a$ posteriori detector. For numerical results, we use mixtures of 4, 8 and 16 Gaussian densities to represent each message. While the complexity is typically higher than the conventional linear estimator, the performance gain may easily justify the additional complexity.

In principle, the proposed scheme is not limited to BPSK modulation and can be generalized to any signal constellation, including QPSK, 8-PSK and 16-QAM. We remark that the algorithm is particularly suitable for BPSK and QPSK modulations. As the constellation size increases, the complexity of the algorithm increases quickly, while the advantage over linear channel estimation vanishes because the interference appears to be more Gaussian-like.

\section{Simulation Results}

In this section, the performance of the $\mathrm{BP}$ algorithm is plotted versus signal-to-noise ratio $S N R=\sigma_{H}^{2} / \sigma_{N}^{2}$. We set $\alpha=.99$, limit the maximum number of Gaussian components to 16 and set the block length $n=200$. Within each block, there is one pilot every 4 symbols.

\section{A. Bit Error Rate (BER) Performance}

The BP algorithm with Gaussian mixture messages described in Section III was simulated. We also simulated two other receivers for comparison. One is denoted by "MMSE", which estimates the desired channel by taking a linear combination of adjacent received value. This MMSE estimator treats the interference as white Gaussian noise. The other is denoted by "ML with full CSI", which performs maximum likelihood (ML) detection symbol by symbol assuming that the realization of the fading processes is revealed to the detector by a genie. It is an ideal receiver and serves as a benchmark or lower bound for any receiver.

Figs. 2(a)-2(c) show the BER performance where the power of the interference is $10 \mathrm{~dB}$ weaker, $3 \mathrm{~dB}$ weaker and equal to that of the desired user, respectively. The BP algorithm generally gives a significant performance gain over the MMSE algorithm, especially in the high SNR region. Note that thermal noise dominates when the interference is weak. Therefore, relatively little performance gain over the MMSE algorithm is observed in Fig. 2(a). In the very low SNR region, the MMSE algorithm outperforms the BP algorithm, which is probably due to the limitation on number of Gaussian components.

The trend of the BP curves show that the BP algorithm works as an interference cancellation receiver rather than as interference suppression. Moreover, the performance gap between BP and genie-aided ML is mainly due to the channel estimation error, which we will investigate next.

\section{B. Channel Estimate Performance}

Fig. 3 shows the mean square error for the channel estimation versus SNR with interference signal $3 \mathrm{~dB}$ weaker than the desired one. As we expect, the channel estimate from the $\mathrm{BP}$ algorithm is much more accurate.

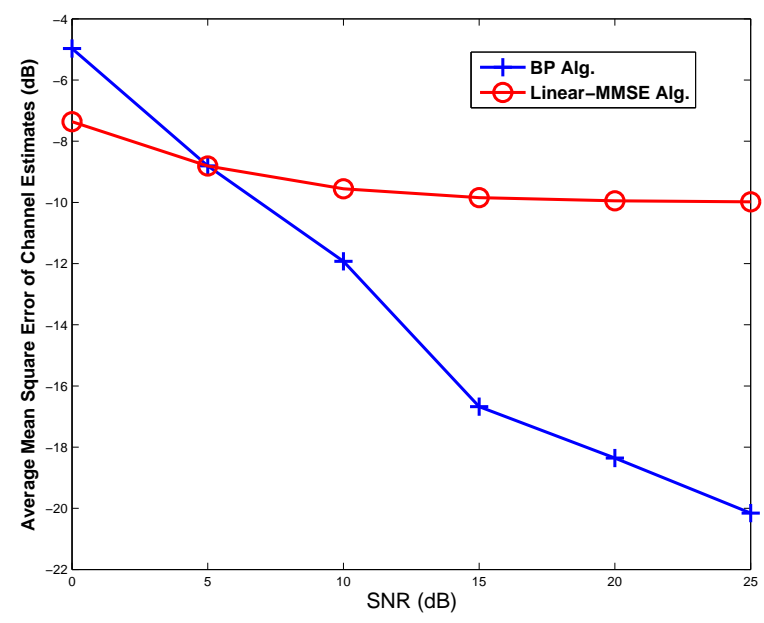

Fig. 3: Channel estimation error with $3 \mathrm{~dB}$ weaker interference.

\section{Impact of Mixture Gaussian Approximation}

As aforementioned, the number of Gaussian components in the exact messages related to the fading coefficients grows exponentially. In order for implementation, we have to truncate or approximate the mixture Gaussian message from time to time. In this paper, we take the simplest way to deal with it: Keep only a fixed number of components with maximum amplitudes. The maximum number of components does have 


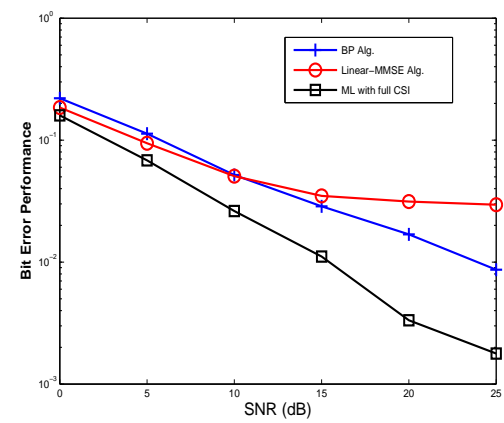

(a)

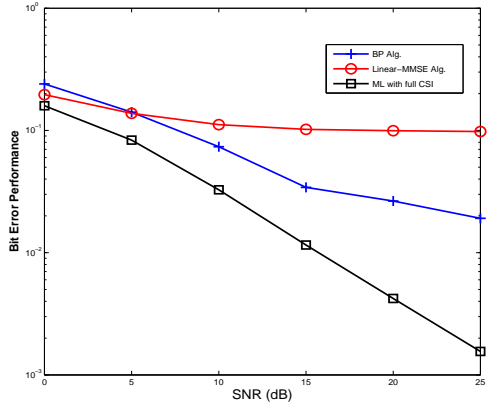

(b)

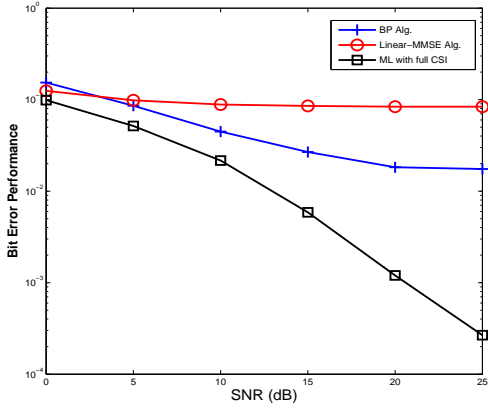

(c)

Fig. 2: BER performance of the BP algorithm. (a) The power of the interference is $10 \mathrm{~dB}$ weaker than that of the desired user. (b)The power of the interference is $3 \mathrm{~dB}$ weaker. (c)The power of the interference is identical to that of the desired user.

some impact on the algorithm. Here, we presents some numerical experiments to illustrate it in more detail.

When the pilot density is high, say $50 \%$, there is no need to keep many Gaussian components in each message. In fact, keeping 2 components is essentially enough. However, when the pilot density is lower, say $25 \%$, the situation is different. Fig. 4 shows the BER performance when we keep different numbers of Gaussian components in the BP algorithm where the pilot density is $25 \%$. For this case, we need 8 components each message passing step. Indeed, the lower the pilot density is, the more we need to keep the Gaussian components in order for the same performance. It is also quite clear that better channel estimation is obtained at symbols closer to pilots than those farther away from pilots. When the pilot density is low, we must keep sufficient number of components, which means to keep sufficient resolution for the message. Roughly speaking, the number of Gaussian components needed is closely related to the number of hypotheses arising from symbols between the symbol of interest and the nearest pilot. The impact of the Gaussian mixture approximation could also be significant. The research on this direction will be included in our future works.

\section{CONClusion}

In this paper, we propose a joint channel estimation and interference cancellation scheme based on graphical model and belief propagation. Unlike conventional linear channel estimation followed by symbol-wise detection, the proposed scheme exploits the non-Gaussian statistics of the interference. By passing locally computed messages, the overall complexity is limited to a constant per symbol. Simulation results show that the BP algorithm has significant performance gain over the traditional interference suppression schemes. Future works will include more improvement of this BP algorithm, and incorporating iterative detection of channel codes in the same message-passing framework.

\section{REFERENCES}

[1] I. Abou-Faycal, M. Médard, and U. Madhow, "Binary adaptive coded pilot symbol assisted modulation over Rayleigh fading channels without

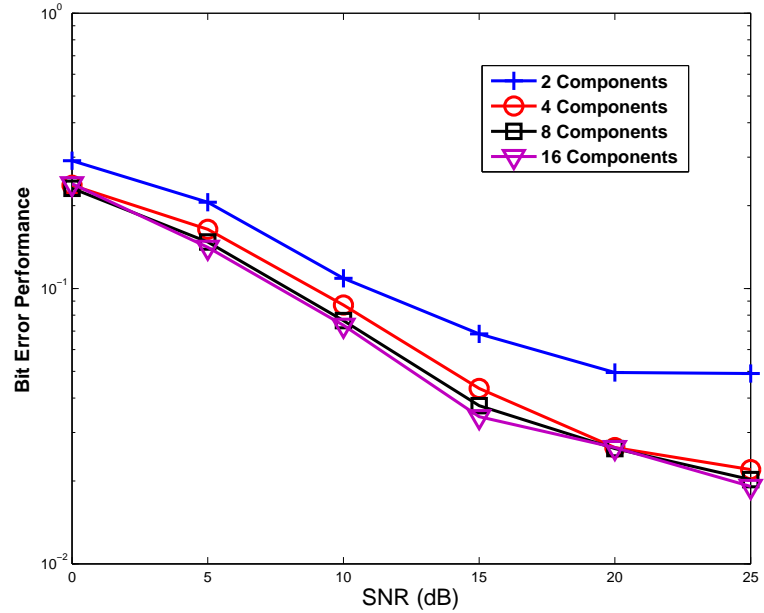

Fig. 4: BER performances with different number of components in the messages. The density of pilot is $25 \%$.

feedback," IEEE Trans. Commun., vol. 53, no. 6, pp. 1036-1046, June 2005.

[2] L. R. Bahl, J. Cocke, F. Jelinek, and J. Raviv, "Optimal decoding of linear codes for minmizing symbol error rate," IEEE Trans. Inform. Theory, vol. 20, no. 2, pp. 284-287, 1974.

[3] A. W. Eckford, "The factor graph EM algorithm: Applications for LDPC codes," in 6th IEEE Workshop on Signal Processing Advances for Wireless Communications (SPAWC), New York, NY, USA, 2005.

[4] T. Kashima, K. Fukawa, and H. Suzuki, "Adaptive MAP detection via the EM algorithm for LDPC-coded MIMO OFDM mobile communications," IEICE Trans. Commun., vol. E90-B, no. 2, pp. 312-322, Feb. 2007.

[5] F. R. Kschischang, B. J. Frey, and H.-A. Loeliger, "Factor graphs and the sum-product algorithm," IEEE Trans. Inform. Theory, vol. 47, no. 2, Feb. 2001.

[6] D. C. MacKay, Information Theory, Inference and Learning Algorithm. Cambridge University Press, 2003.

[7] T. J. Richardson, M. A. Shokrollahi, and R. L. Urbanke, "Design of capacity-approaching irregular low-density parity-check codes," IEEE Trans. Inform. Theory, vol. 47, no. 2, pp. 619-637, Feb. 2001.

[8] T. J. Richardson and R. L. Urbanke, "The capacity of low-density paritycheck codes under message-passing decoding," IEEE Trans. Inform. Theory, vol. 47, no. 2, pp. 599-618, Feb. 2001.

[9] K. Sil, M. Agarwal, D. Guo, and M. L. Honig, "Performance of Turbo decision-feedback detection and decoding in downlink OFDM," in IEEE Wireless Commun. \& Networking Conf., 2007. 